\title{
Multiple Trips Pattern Mining
}

\author{
Riaz Ahmed Shaikh \\ Department of Computer Science \\ Shah Abdul Latif University \\ Khairpur, Sindh, Pakistan
}

\author{
Kamelsh Kumar \\ Department of Computer Science \\ Sindh Madressatul Islam University \\ Karachi, Sindh, Pakistan
}

\author{
Rafaqat Hussain Arain \\ Department of Computer Science \\ Shah Abdul Latif University \\ Khairpur, Sindh, Pakistan \\ Hidayatullah Shaikh \\ Department of Computer Science \\ Shah Abdul Latif University \\ Khairpur, Sindh, Pakistan
}

\author{
Imran Memon \\ College of Computer Science \\ Zhejiang University, Hangzhou \\ Zhejiang, 310027, China \\ Safdar Ali Shah \\ Department of Computer Science \\ Shah Abdul Latif University \\ Khairpur, Sindh, Pakistan
}

\begin{abstract}
In recent years, photograph sharing is one of the most mainstream web service, for example, Flickr, trip advisor and numerous other web services. The photograph sharing web services give capacities to include Geo coordinates, tags, and user ID to photographs to make photograph organizing easily. This study focuses on Geotagged photographs and discusses an approach to recognize user multiple trips pattern, i.e., common arrangements of visits in towns and span of stay and also elucidating labels that describe the multiple trips pattern. First, we segment collection of photos into multiple trips and categorize the photos manually based on photo trips into multiple trips, themes such as Landmark, Nature, Business, Neutral and Event. Our method mines multiple trips pattern for multiple trips theme categories. The experimental result of our technique beats other methods and accurate segmentation of photo collections into numerous trips with the $85 \%$ of accuracy. The multiple trips categorize about $91 \%$ correctly using tags, photo id, titles of digital photos, user id and visited cities as features. In last, we demonstrate the motivating examples showing an application with which one can find multiple trips pattern from our datasets and other different queries visit duration, destination and multiple trips' theme on trips.
\end{abstract}

Keywords-Multiple trips pattern mining; multiple trips classification; geo-tagging

\section{INTRODUCTION}

In recent years, there have been great innovations of digital camera and camera phone to sharing digital photos assigned tags, time stamps, geographical reference and visual information on web services such as Flickr, Facebook, Picasa and Panoramio and many other websites. Most popular internet application is a social networking service; millions of users share their information on these web services [1]-[5]. Users share their travel experience like photo and video of these social media web services. Photo sharing web services comprise billion of images accessible everywhere taken on earth. Increases volume of these images is defined various forms, including Geo tagged information, photographs, time and other variety of textual information. Increase volume of
Geo-reference, social media resource such as photos and videos document together with Geo tagged facilities. Textual metadata and temporal references; these enhanced the multimedia provided wealth data to solve the vision and media task. To discover graphic related information, knowledge about human societies and open new opportunities provide by multimedia. In computer vision research works most of people at single location make rich signified from image and also recognize image where an image was taken as well as image contents [6]-[12]. In this paper, we downloaded 10 million, data from Flickr using public Flickr API. This method automatically segments photo collection every user into multiple trips and also categorized multiple trips into the multiple trip's theme.

Following is our contribution in this paper:

- Our proposed algorithm empowers the novel knowledge detection, several trips pattern that depends on users' experiences, from the collection of Geotagged photos.

- We performed experiments for the performance evaluation of our proposed algorithm. The experimental result of our technique beats other methods and accurate segmentation of collection of photos into numerous trips with the $85 \%$ of accuracy. The multiple trips categorize about $91 \%$ correctly using tags, photo id, titles of digital photos, user id, and visited cities as features.

Table I used to outline the differences between our work and other significant studies. Here we show some essential issues about mining user trip and recommendation system, including: location Query Locations (LQ), timestamp (TS), GPS coordinates longitude/latitude (GPSC) and whether it considers the following ideas: Title assign to photo (TP), user travelling sequences (UTS), Trip classification (TC), categorization (CT), Visiting Time (VT) Recommendation system (RS), Geo tagged photo (GTP), user preference (UP), Distance (DI) sun database (SDB), Automatic detection (ATD) [3]-[18]. 
TABLE I. DIFFERENCES BETWEEN OUR WORK AND EXISTING WORKS

\begin{tabular}{|c|c|c|c|c|c|c|c|c|c|c|c|c|c|c|}
\hline & Oִ & 2 & 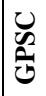 & in & $\stackrel{5}{5}$ & 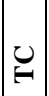 & 七 & 5 & $\approx$ & 氜 & $\hat{s}$ & $\overline{\mathbf{\theta}}$ & के & 量 \\
\hline Zheng Liu et al. 2012 & $\bullet$ & $\bullet$ & & $\bullet$ & & & $\bullet$ & & $\bullet$ & $\bullet$ & & $\bullet$ & & $\bullet$ \\
\hline Takeshi et al. 2013 & & & $\bullet$ & $\bullet$ & $\bullet$ & & & & $\bullet$ & $\bullet$ & $\bullet$ & - & & \\
\hline Platt et al. 2002 & $\bullet$ & $\bullet$ & $\bullet$ & & & & $\bullet$ & $\bullet$ & & & & & & \\
\hline Toshihiko et al. 2013 & $\bullet$ & & & $\bullet$ & & & & $\bullet$ & - & $\bullet$ & $\bullet$ & - & & \\
\hline Jianxiong et al. 2010 & & $\bullet$ & $\bullet$ & & & & $\bullet$ & & $\bullet$ & & & $\bullet$ & - & - \\
\hline $\begin{array}{l}\text { Yan-Ying Chen et al. } \\
2013\end{array}$ & $\bullet$ & & & $\bullet$ & $\bullet$ & & $\bullet$ & & $\bullet$ & $\bullet$ & $\bullet$ & - & & - \\
\hline David et al. 2009 & $\bullet$ & & $\bullet$ & $\bullet$ & & & & & $\bullet$ & $\bullet$ & & & & \\
\hline Zheng et al. 2012 & & $\bullet$ & $\bullet$ & & $\bullet$ & $\bullet$ & $\bullet$ & $\bullet$ & $\bullet$ & $\bullet$ & & & & $\bullet$ \\
\hline Richang et al. 2010 & & $\bullet$ & $\bullet$ & & & & $\bullet$ & & & $\bullet$ & & & & \\
\hline Claudiu S et al. 2010 & & & $\bullet$ & $\bullet$ & & & $\bullet$ & & $\bullet$ & $\bullet$ & & & & $\bullet$ \\
\hline Yue Shi et al. 2013 & - & & $\bullet$ & $\bullet$ & & & $\bullet$ & & $\bullet$ & $\bullet$ & & - & & $\bullet$ \\
\hline Adrian et al. 2009 & & & $\bullet$ & $\bullet$ & & & $\bullet$ & & & $\bullet$ & $\bullet$ & & & $\bullet$ \\
\hline Zheng et. al. 2011 & $\bullet$ & & $\bullet$ & $\bullet$ & - & & & - & $\bullet$ & $\bullet$ & $\bullet$ & - & & \\
\hline This Paper & $\bullet$ & & $\bullet$ & $\bullet$ & & $\bullet$ & $\bullet$ & $\bullet$ & $\bullet$ & $\bullet$ & $\bullet$ & - & & - \\
\hline
\end{tabular}

\section{PROBLEM DEFINITION}

\section{A. Characteristics of Photo Trajactory}

We utilize Geo tagging photographs in light of the fact that it is extremely rough than GPS trajectories. GPS trajectories acquire by GPS receiver through portable devices. You can see various users take photos during their movements because users take photos only when they see some attractive or interesting thing, in general users do not upload unattractive photos on web and various users visited different locations within the city. This is a great advantage of multiple trips mining because users take photos visiting different locations during their trips. The large data available on photo sharing web services, but it is hard to collect data from GPS trajectories to group of users.

\section{B. Dataset}

We downloaded photo collections of crawled users using Flickr public API and arranged data set such as time, user id, Geo coordinates, title and tags assigned to Geo tagged photos. Single photo assigned multiple tags at that time also possible same tag allocated to several photos.

We model the photo sets as $\mathbb{p} \triangleq\{p\}$ where $p$ has characteristics of time captured photot $p_{p}$, the ID of owner $u_{p}$ location captured denoted by geo coordinate and assign title to photos. According to above description the photo collections each user represented by $\mathbb{P}_{\mathrm{u}} \subseteq \mathbb{P}$, where all photos $\mathrm{p} \subseteq \mathbb{P}$ are acquired by the similar user with the user ID $u_{p}$ arranged in sequence that is $\mathbb{P}_{\mathbf{u}}$ can as temporal and spatial order.

We separated tags from Geo tag photos; use the variable $\ell$ to represent by the tag and $\mathcal{L}$ to represent by all set of tags. Each tag assigned multiple photos and multiple photos have assigned similar tag. We representation $\mathcal{L}_{\mathrm{s}}$ to set of tags appear in any subset $\mathbb{p}_{s} \subseteq \mathbb{p}$ of photo. The subset of photo related with particular $\operatorname{tag} \mathbb{P}_{1} \subseteq \mathbb{P}$. Photo associate with tags in the subset of the particular tag represent by $\mathbb{P}_{,, \ell}$. The users set related to photos in $\mathbb{P}_{,}, \ell$ and $\mathrm{n}_{\mathrm{s}}$ as set of all users related to photos in $\mathbb{P}_{s}$.

\section{The Data Model for Multiple Trips Pattern}

Elementary elements for photos $\mathbb{P}$ and $\operatorname{tags} \mathcal{L}$, the trips formally define as:

$$
\mathcal{P} \mathcal{T}=\varepsilon, \mathcal{T}, \mathbb{L}, \mathcal{A}
$$

Due to above equation the element defines as $\varepsilon$ represented by locations sequence of visits user. Locations have hierarchy, such as scene, city, province and country. Our study focuses on the city level. Further, $\varepsilon=\left(\mathcal{C}_{1, \ldots \ldots . . .,} \mathcal{C}_{\mathrm{n}}\right)$ represented cities sequences visited by users. $\mathcal{T}=\left(\mathcal{T}_{1, \ldots . ., \mathcal{T}_{\mathrm{n}-1}}\right)$ represented visited sequence duration determined by two consequences cities. Then, $\mathcal{P}=\mathcal{P}_{\mathcal{C} 1, \ldots \ldots .,} \mathcal{P}_{\mathcal{C} \text { n }}$ represented set of photo captured in visited cities and $\mathbb{L}=\left(\mathcal{L}_{\mathrm{c} 1, \ldots \ldots,} \mathcal{L}_{\mathrm{cn}}\right)$ represented tags assign to photos. Finally, $\mathcal{A}$ represented multiple trip's theme, users are chief object on the trips determined. Fig. 1 demonstrates the multiple trips of user which contain three trips 1) visiting famous landmarks in China, 2) everyday gathering with friends in Germany, 3) and last shopping in Paris. We aim to detect multiple trips pattern on each trip into multiple trips themes. As the frequently visited cities and duration of visiting and characteristic of tags order for multiple trip pattern. We describe the multiple trips, tag and characteristic trips pattern, tags denoted what to enjoy and what to see during to multiple trips pattern [19]. Content analysis using shape and image edge detection are useful techniques for object recognition [20], [21].

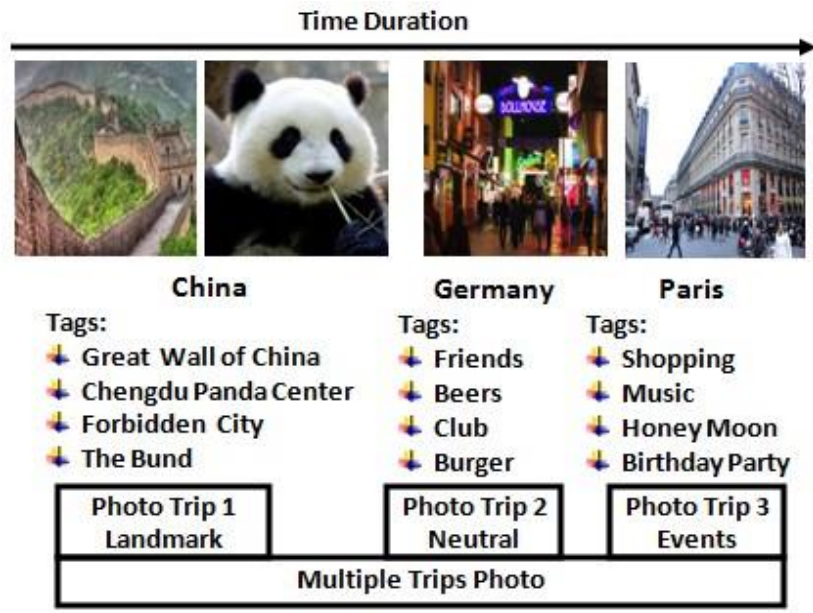

Fig. 1. User's multiple trips photo.

\section{APPROACH}

The idea to detect event from photo collections is to use gaps of capture time duration because the information about the location of photos did not available at that time. The peoples' movement reflects directly transition of photo capture location and also necessary to efficiency improve event detection. The Geo-tagged photos each tag assigns well define the trip. The user assigns similar labels on groups of successive photos [1] which ought to uphold photo collections algorithm of segmentation. We can use to capture the location and time gaps, tag on segmentation of photo collections. In [3] proposed algorithm separate photo into the event. It detects the time, which regarded the event changes. The algorithm first sort photo time captured if the change time gaps between two 
photos, then sorted list as change event consider if it's much higher than local gap:

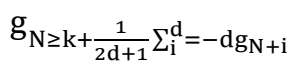

The appropriate threshold is $\mathrm{K}$ and " $\mathrm{d}$ " is a size of window. If $\mathrm{N}+\mathrm{i}$ to photograph past the end of photo collection, expression is overlooked and the denominator $2 d+1$ is decreased for each disregarded term to keep the average

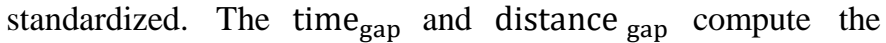
distance and transition time of two successive photos. We calculate the gaps between two consecutive photos: $\mathrm{p}_{\mathrm{k}}$ and $\mathrm{p}_{\mathrm{k}+1}$ show the distance and time gaps following equation:

$$
\begin{gathered}
\text { distance }_{\text {gap }}=\log (\mathrm{D} \emptyset) \\
\text { time }_{\text {gap }}=\log \left(\mathrm{t}_{\mathrm{pk}+1}-\mathrm{t}_{\mathrm{pk}}\right)
\end{gathered}
$$

Finally, the gap $g_{N}$ Is considered as changing trip when much higher the local gap average calculates by

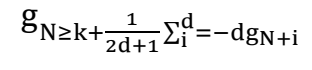

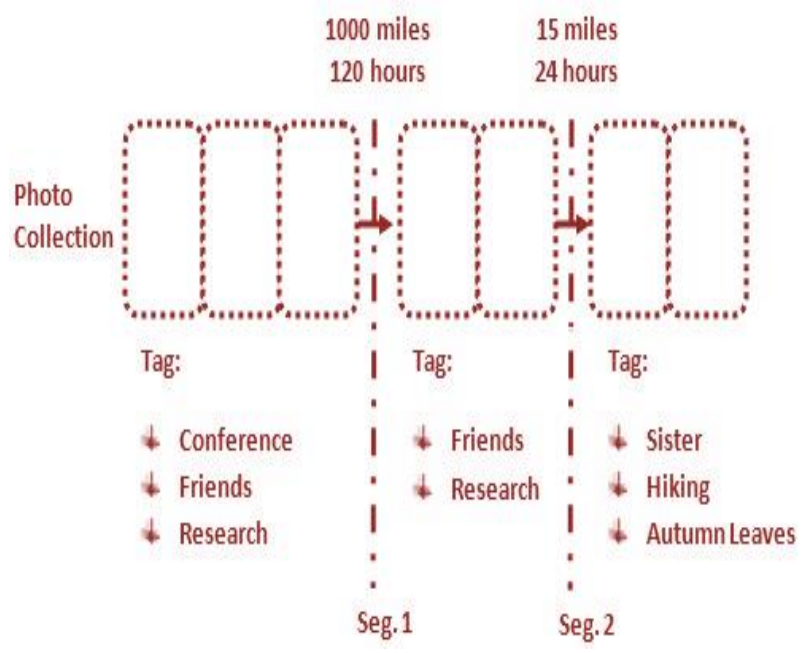

Fig. 2. Example of photo collection segmentation.

In Fig. 2 shown the example where we consider photo collections containing of photo capture when attending conference and user research in the lab with their friends. Our algorithm of segmentation collection as Seg.1; the large gaps caught time and area while the labels are comparable with taking after photograph. In Seg.2, segmentation tags allocated to photographs in the final group did not share labels to previous photos.

\section{PROPOSED Model: THE Multiple TRIPS CLASSIFICATION}

Proposed model is very adequate for the multiple trips classification, after segmentation of photograph collection and connected with city names and duration of visit to various trips we characterize numerous trips as indicated by different trip's theme. The numerous trips theme empowers clients to find various trip patterns as per their objectives on the trip, and distinct theme result's trip in dissimilar descriptions and patterns, despite the fact that we visit the same sites.

\section{A. Multiple Trips Theme}

We surveyed various websites of travel forums, agencies and blogs regarding travel experience. On the basis of our survey we categorize various trips in five key objectives.

Landmark: Visit of popular sightseeing, world heritage, for example, the Great Wall of China, Chengdu Panda center, etc. as shown in Fig. 1.

Nature: Visit of such places which are popular for rich nature, for exp. Tiananmen Square in Beijing and Beihai parks, to communicate with nature.

Event: Visit for attending events, like Ninfeng in Shanghai and a wedding ceremony.

Neutral: Everyday small trips to visit adjacent areas for example, gatherings with friends and relatives at hotel or bar, and to enjoy delicious food with family such as famous hotspots in Chengdu.

\section{Business: To visit places for the business purpose.}

Note that we collected multiple trips only those users who have visited more than three trips. We describe these five types of themes of trip and develop an algorithm to categorize various trips into theme of multiple trips. We ought to note that user's trips going to the similar areas may belong with different classes, since individuals go to the similar areas with distinctive aims

\section{B. Classifier}

For the classification of multiple trips Support Vector Machines (SVMs) has been used. To prepare support vector machine we assigned labels manually to 20000 randomly selected various trips photographs by their multiple themes of trips. We categorize various trips into five classes and prepare a SVM [15] for each of the various themes of trip. To carry out numerous trips photograph categorization on a set of test, we execute five classifiers on different trips and pick the classifications with most elevated score (most prominent optimistic separation from the support vector machines isolating hyperplane). We performed separation of the labeled various trips into partitioning so as to prepare and testing sets various tours of the same clients so as to evade the likelihood that related numerous trips of the similar individual show in training sets.

\section{EVALUATION}

10 million Geo-tagged photographs are downloaded from Flickr website. The several tags allocated to the photographs are 82.9 million whereas the quantity of novel tags between them is 4.7 million. Quantity of photo proprietors is $79.7 \mathrm{~K}$, between the $49.1 \mathrm{~K}$ clients whose home town areas are accessible (on Flickr, clients can enlist their locations), around $18 \%$ of photographs are caught inside of clients' city, around $60 \%$ of photographs are caught inside of clients' country, $22 \%$ of photographs are caught outside the county of client. As the sizes of towns are not stable, we can't straightforwardly state that all $17 \%$ of photographs were caught around homes of the client. Although, the measurements demonstrate that the users are capturing photographs in different areas, which incorporates both places adjacent and abroad. In this way, we 
hope to have the capacity to recognize a various trips pattern from the collection of photographs.

\section{A. Segmentation Accuracy}

In this section, we examined an accuracy of segmentation of the collections of photographs and the effect of the tags and distance/time gaps.

\section{B. Evaluation Metrices}

In this section, we compare segmentation algorithm with existing algorithms (Platt el at 2002) and three differences of our proposed algorithm which are given below: 1) suppose only the distance gaps, 2) one tag only, 3) supposes captured time and distance gap both $(\beta=0$ parameter) in the sequence to contribute from every element. During experiment, set parameter such as $(\mathrm{d}, \mathrm{k}, \alpha, \beta)$ of these methods are the best as performance in a different training set of photographs. The fact is, we arbitrarily select eighteen clients who seem like active clients of Flickr and physically divided their photograph collection into several trips. Least number of testing user's about 2240 and highest number of test user's is about 5022 with the aggregate number of test user's is 115208 .

We use compare metrics in existing work of occasion clustering for collections of photo [18], [19], following equations is for precision and recall of detecting boundaries for event.

$$
\text { Precision }=\frac{\text { no.of corectlydet ectedboundaries }}{\text { Total No.of det ectedboundaries }}
$$

$$
\text { Re call }=\frac{\text { no.of corectlydet ectedboundaries }}{\text { Total No.of det ectedboundaries }}
$$

The trade-off among recall and precision is that lower recall end to result in high precision and vice versa.

The high precision joins some real truth diverse trips into several trips and high call tends to over segmentation for the collections of photo. To maintain balance between the recall and precision is vital. $F$ measurement $(\mathrm{F} 1)$ is the harmonic mean of recall and precision, (3) used for the assessment of balance between precision and recall. The range of values is from 0 to 1 for F1, whereas a higher value is good.

$$
F 1=\frac{2(\text { precision })(\text { recall })}{(\text { precision })+(\text { recall })}
$$

\section{Results and Discussions}

Table II shows the result of photo collections segmentation. To over the segment photo collections means higher recall and lower precision values Platt's algorithm results show that segmented photo collection into a smaller piece of the trip. On the other side, tend to combine various trips into multiple trips the algorithm count distance gaps only and higher precision lower recall resulting shows us. The based on a distance gap algorithm and plats algorithm; an algorithm considers both distance gap and time gap. The algorithm outperforms the other algorithm by considering the title and assign tags to photos resulting shows F1 best. It turns out complement other algorithm, the performance of tags counting algorithm were poor. By itself, the many tags on the photographs are insufficient for segmentation. Being merge with different frameworks the tags offer additional advantage to them, since straightforwardly reflect what is going on in the photographs.

TABLE II. SEGMENTED Photo COLLECTION

\begin{tabular}{|l|l|l|l|}
\hline Methods & Precision & Recall & F1 \\
\hline Tag & 0.41 & 0.76 & 0.58 \\
\hline User id & 0.32 & 0.69 & 0.43 \\
\hline Time & 0.67 & 0.87 & 0.76 \\
\hline Distance and Time & 0.69 & 0.83 & 0.76 \\
\hline Distance & 0.74 & 0.38 & 0.50 \\
\hline Our proposed method & 0.79 & 0.91 & 0.85 \\
\hline
\end{tabular}

The spatial feature based matrix of confusion is shown in Table III.

TABLE III. Spatial FeAture BASEd Confusion Matrix (\%)

\begin{tabular}{|l|l|l|l|l|l|}
\hline & $(\mathbf{L a})$ & $(\mathbf{N})$ & $(\mathbf{E})$ & $(\mathbf{N E})$ & $(\mathbf{B})$ \\
\hline Landmark & 50.2 & 21.4 & 9.7 & 3.5 & 11.7 \\
\hline Nature & 3.0 & 28.5 & 2.3 & 2.0 & 3.3 \\
\hline Event & 2.2 & 1.2 & 18.1 & 1.2 & 5.5 \\
\hline Natural & 44.0 & 48.4 & 71.0 & 92.7 & 60.2 \\
\hline Business & 0.60 & 0.26 & 0.1 & 0.5 & 19.2 \\
\hline
\end{tabular}

\section{CONCLUSIOIN AND FUTURE WORK}

The described idea of multiple trips and proposed multiple trips pattern mining algorithms that detected novel multiple trips from the collection of Geo-tagged photos on web. First photo collections can be dividing into multiple trips by segmentation and categorize them multiple trip's theme. After this identify several trips patterns as arrangements of most often traveled areas and their visit spans. Mine labels, tolerating their geological scope to incorporate a delineation of a various trip pattern with the objective that clients interpret them as what to expect and see if grasp the patterns. Various trips arrangement, results demonstrate that texture components of digital photos, i.e., titles and tags of photos are the very competent components and spatial components of users' trips, such as visited towns, can supplement the textural component. Utilizing these elements can categorize around $91 \%$ of numerous trips accurately. In future work different ANN classifiers may be used.

\section{REFERENCES}

[1] Crandall, D., J., Backstrom, L., Huttenlocher, D., Kleinberg, J., "Mapping the world's photos", International Conference on World Wide Web, pp. 761-770, 2009.

[2] Zheng, Y., T., Zha, Z., J., Chua, T., S. "Research and applications on Geo-Referenced multimedia a Survey" Multimedia Tools and Applications, Vol. 51(1), pp. 77-98, 2010.

[3] John C. Platt, M., C., Brent A., F., "Photo TOC: Automatic Clustering for Browsing Personal Photographs", Information, Communications and Signal Processing, pp. 1-5, 2003. 
[4] Zheng, Y., T., Zha, Z., J., Chua, T., S., "Mining Travel Patterns from Geo-Tagged Photos", ACM Transactions on Intelligent Systems and Technology, Vol. 3(3), pp. 1-18, 2012.

[5] Xiao, J., Hays, J., Ehinger, K., A., Oliva, A.,; Torralba, A., "SUN database: Large-scale Scene Recognition from Abbey to Zoo", Computer Vision and Pattern Recognition (CVPR), pp. 3485-3492, 2010.

[6] Yan-Ying Chen, A., J., C., Winston, H., H., "Travel Recommendation by Mining People Attributes and Travel Group Types from CommunityContributed Photos", IEEE Transactions on Multimedia, Vol. 15(6), pp. 1283-1295, 2013.

[7] Liu, Z., Yan, H., Han, H., "Mining Large-Scale Social Images with Rich Metadata and Its Application", Journal of Software, Vol. 7(4), pp. 749756, 2012.

[8] Yamasaki, T., Gallagher, A., Chen, T., "Personalized Intra- and InterCity Travel Recommendation Using Large-Scale Geotags", GeoMM '13 Proceedings of the 2nd ACM international Workshop on Geotagging and its applications in multimedia, pp. 25-30, 2013.

[9] Shi, Y., Serdyukov, P., Hanjalic, A., Larson, M., "Nontrivial landmark recommendation using geotagged photos", ACM Transactions on Intelligent Systems and Technology, Vol. 4(3), 2013.

[10] Claudiu, S., Firan, M., G., Nejdl, W., Paiu, R., "Bringing Order to your Photos: Event-Driven Classification of Flickr Images Based on Social Knowledge", CIKM'10, pp. 189-198, 2010.

[11] Popescu, A., Grefenstette, G., Moëllic, P., "Mining Tourist Information from User-Supplied Collections", Proceedings of the $18^{\text {th }}$ Conference on Information and Knowledge Management, pp. 1713-1716, 2009.

[12] Hong, R., Li., G., Nie, L., Tang, J., Chua, T., "Exploring Large Scale Data for Multimedia QA an Initial Study", CIVR '10 Proceedings of the ACM International Conference on Image and Video Retrieval, pp. 7481, 2010.
[13] Kurashima, T., Iwata, T., Irie, G., Fujimura, K.,. "Travel Route Recommendation Using Geo-tagged Photos", Knowledge and Information Systems, Vol. 37(1), pp. 37-60, 2012.

[14] Giannotti, F., Nanni, M., Pedreschi, D., "Efficient Mining of Temporally Annotated Sequences", SAC '06 Proceedings of the 2006 ACM symposium on Applied computing, pp. 593-597, 2006.

[15] Tsochantaridis, I., Hoffman, T., Joachims, T., Altun, Y., "Support Vector Machine Learning for Interdependent and Structured Output Spaces", Proceedings of the 21st International Conference on Machine Learning, Banff, pp. 104, 2004.

[16] Hao, Q., Cai, R., Wang, X., Yang, J., Pang, Y., Zhang, L., “Generating Location Overviews with Images and Tags by Mining User-Generated Travelogues", MM '09 Proceedings of the 17th ACM international conference on Multimedia, pp. 801-804, 2009.

[17] Rattenbury, T., Naaman, M. "Methods for Extracting Place Semantics from Flickr Tags", ACM Transactions on the Web, Vol. 3(1), 2009.

[18] Cooper, M., Foote, J., Girgensohn, A., Wilcox, L., "Temporal Event Clustering for Digital Photo Collections", ACM Transactions on Multimedia Computing, Communications, and Applications (TOMM), Vol. 1(3), pp. 269-288, 2005.

[19] Alexander, C., Loui, S., M., Savakis, A., "Automated Event Clustering and Quality Screening of Consumer Pictures for Digital Albuming", IEEE Transactions on Multimedia, Vol. 5(3), pp. 390-402, 2003

[20] Riaz Ahmed Shaikh, Jian-Ping Li, Asif Khan, Kamlesh Kumar, "Content Analysis Using Shape and Spatial Layout with Markov Random Field", Indian Journal of Science and Technology, Vol 9(7), pp. 1-6, February 2016.

[21] Kamlesh Kumar, Jian-Ping Li, Saeed Ahmed Khan, "Image Edge Detection Scheme Using Wavelet Transform", International Conference on Wavelets Active Media and Information Processing, vol. 11, pp. 261256, December. 2014. 